\title{
Australian general practitioners views and practices in the management of bacterial vaginosis: A pilot qualitative study
}

\author{
Joyce Toh ${ }^{1}$, Meredith J Temple-Smith ${ }^{1}$ and Jade E Bilardi ${ }^{1,2 *}$ \\ ${ }^{1}$ Department of General Practice and Primary Health Care Academic Centre, University of Melbourne, 200 Berkeley Street, Parkville, 3010, Victoria, Australia \\ ${ }^{2}$ Melbourne Sexual Health Centre, Central Clinical School, Monash University, 580 Swanston Street Carlton, 3053, Victoria, Australia
}

\begin{abstract}
Introduction: Bacterial vaginosis $(\mathrm{BV})$ is the most common vaginal infection among women of reproductive age. Recent research shows women often feel dissatisfied with the management of BV in general practice. Few data are available on general practitioners (GPs) management of BV in Australia. This study explored GPs views and practices in the management of BV.
\end{abstract}

Methods: A pilot qualitative study was undertaken in which eight GPs were purposively recruited to participate in one-on-one semi-structured interviews. Interviews were recorded, transcribed verbatim and analysed thematically.

Results: GPs did not consider BV a serious condition, had poor levels of knowledge around BV-related sequelae and risk factors, used varying and inconsistent diagnostic and treatment practices, and rarely asked women about self-management of symptoms. Most GPs recognised BV could have some psychosocial impact on women's lives; those with an interest in sexual health tended to be more empathetic. Major barriers to the improved management of BV in general practice included a lack of time, a lack of training and knowledge, and discomfort discussing sexual health issues particularly among male GPs.

Conclusion: Past research has shown women want more sensitive and consistent management of BV in general practice. BV management in general practice could be enhanced through improved awareness of BV-related risk factors and sequelae and women's use of self-help remedies. Women need well-informed, evidence-based advice around optimal management and risks for BV.

\section{Introduction}

Bacterial vaginosis (BV) is the most common vaginal infection among women of reproductive age, affecting $10-30 \%$ of women in developed countries $[1,2]$. While it remains unclear whether BV is a sexually transmitted infection (STI), there is strong evidence that sexual activity is associated with an increased risk of BV. Sexual risk factors include penile-vaginal sex, a female sexual partner with BV, multiple male sexual partners and lack of condom use, and risky lifestyle practices including vaginal douching [2-4]. Adverse sequelae associated with BV include pre-term labour, miscarriages, low-birth weight $[5,6]$, and an increased risk of HIV and other STIs [7,8]. Previous studies have also shown BV has significant psychosocial impacts on women's quality of life, in particular their self-esteem and sex lives [9].

Current Australian STI management guidelines [10] recommend the work-up for women presenting with vaginal discharge include history-taking, physical examination and a high vaginal swab (HVS) for collection of specimens for microscopy and gram-staining. Diagnosis of BV is based on the presence of at least three out of four Amsel criteria, including clinical findings of a thin, white, homogenous discharge, vaginal $\mathrm{pH}>4.5$, a positive whiff test and clue cells on saline wet mount [11]. Metronidazole remains first-line for BV management in non-pregnant patients [11].

Past studies have shown that primary care physicians generally have poor levels of knowledge around BV-related risk factors and physical sequelae, seldom follow the "classic approach" [12] for diagnosing vaginal symptoms [13-15], infrequently use Amsel criteria [15] and often treat vaginal symptoms empirically [16]. Clinicians also often view BV as a trivial condition [17] and do not recognise the psychosocial impact it can have on women's lives [9]. Women's concerns with BV management in primary care include GPs' poor knowledge of its aetiology, disparities in management advice and insensitive attitudes [18]. Data on primary care management of BV in Australia is lacking. The aim of this pilot study was to explore Australian GPs' knowledge and practices in the management of $\mathrm{BV}$, to inform and enhance care of this condition.

\section{Methods}

A qualitative descriptive (QD) approach was used to explore GPs' knowledge and practices in the management of BV. QD is a pragmatic approach, useful for exploratory healthcare studies with limited time and resources, aimed at gaining knowledge around a topic of clinical interest [19]. GPs were purposively recruited between March and May 2017 to participate in semi-structured interviews. They were recruited via the Department of General Practices' (University of Melbourne) Victorian Primary Care Practice-based Research Network (VicReN)

${ }^{*}$ Correspondence to: Jade Bilardi, Melbourne Sexual Health Centre, 580 Swanston Street, Carlton, Victoria 3053 Australia, Tel: +61 39035 5511; Email: jbilardi@mshc.org.au

Received: April 08, 2018; Accepted: April 18, 2018; Published: April 20, 2018 
and snowball sampling methods. We aimed to recruit a diverse range of GPs including males and females of various ages and clinical experience, working in metropolitan and regional/rural areas, and with and without a stated interest in sexual health. To be eligible, GPs had to be working in general practice in Victoria, possess good comprehension of spoken and written English and be willing to voluntarily participate in an interview which would be audio-recorded. Ethics approval for this study was granted by the Department of General Practice Human Ethics Advisory Group, the University of Melbourne, ID 1647908.1.

\section{Data collection}

The interview schedule was piloted with a medical student and two GPs, resulting in minor wording changes. Semi-structured oneon-one interviews were conducted at the University of Melbourne, at participants' clinics or by telephone. Participants were provided with a plain language statement and gave verbal or written consent to participate. GPs were asked 15 demographic questions, followed by a series of semi-structured questions on their views and knowledge of BV, past training, diagnostic and management practices and barriers to care.

\section{Data analysis}

Weekly meetings were held with the research team to reflect on the sampling framework, interview schedule and preliminary data analysis and to allow further exploration in subsequent interviews. Interviews were recorded, transcribed verbatim and analysed thematically. Each transcript was read multiple times, coded and categorised into broader themes and sub-themes by JT. A block and file approach [20], whereby segments of transcripts were slotted under broader themes and subtheme headings in a table, was used to categorise data. Themes were derived both deductively from the interview schedule questions and inductively from independent themes recurring in the data itself. A sub-set of interview transcripts were independently reviewed by JB and MTS to confirm coding and themes. No major differences around major themes and sub-themes were evident between researchers.

\section{Results}

Of 13 GPs who indicated interest in or were referred to the study, eight participated. One GP who expressed interest could not be reached, one indicated interest but did not respond to further correspondence, and three who were referred to the study did not respond to the invitation. Interviews ranged from 20-30 minutes. Six interviews were conducted face-to-face at the University of Melbourne and two via telephone. Participants' demographic characteristics are outlined in Table 1.

Three main themes were identified in the interview data: GPs views and knowledge, actual practice and barriers to care.

\section{Views and knowledge}

\section{Not medically serious and poor knowledge}

It was common for GPs to think BV was not a serious medical condition and that there were no adverse physical sequelae or pregnancy only related sequelae associated with $\mathrm{BV}$, such as miscarriage or preterm delivery.

No, no it's not serious at all. It can [have] implications of involvement in miscarriage and during pregnancy but we're really talking about outside of that (GP4, female).

While almost all GPs were aware of some risk factors associated with BV, one GP did not think there were any.
Table 1. Demographic and practice characteristics of participants $(\mathrm{N}=8)$.

\begin{tabular}{|l|l|l|}
\hline & N & Mean \\
\cline { 1 - 2 } & [Range] \\
\hline Age & & $40[27-59]$ \\
\hline Sex & & \\
\hline Male & 3 & \\
\hline Female & 5 & \\
\hline Practice location & & \\
\hline Metropolitan Victoria & 7 & \\
\hline Rural/regional Victoria & 1 & \\
\hline Country medical degree was obtained & & \\
\hline Australia & 2 & \\
\hline United Kingdom & & \\
\hline Training level & 6 & \\
\hline GP fellow & 2 & \\
\hline GP registrar & & $13[1-33]$ \\
\hline Number of years working in general practice & & $10[3-18]$ \\
\hline Number of GPs working at practice & & \\
\hline Number of GPs with more than 50\% of patients being & & \\
\hline females of reproductive age & & \\
\hline Male & & \\
\hline Female & & \\
\hline Self-stated interest in sexual health & & \\
\hline Male & & \\
\hline Female & & \\
\hline Specialist training in sexual health & & \\
\hline Number of women they diagnosed with BV each year & & \\
\hline & & \\
\hline
\end{tabular}

I didn't think there are any particular risk factors...no I actually say it's pretty bad luck, I'm not aware of any particular things that are known to predispose (GP4, female).

Most GPs were unaware BV is associated with an increased risk of STIs and HIV. While GPs recognised that sexual activity may be a risk factor, they did not specify which sexual practices are strongly associated with BV.

...changes in sexual partners would be the one that I mainly think of, otherwise I'm not too sure about others (GP6, male).

When prompted, most GPs recognised vaginal douching as a lifestyle risk factor for BV. However, some seemed unaware of this risk and assumed they were being asked about the benefits of douching when asked what they would advise women regarding this practice.

I'm not aware that there's any evidence that supports that as being a treatment (GP2, male).

In addition to a lack of knowledge on specific risk factors associated with BV, many GPs, especially those without an interest in sexual health, seldom asked women about their use of self-help remedies. GPs with an interest in sexual health were more likely to ask women about self-help remedies and advise against their use.

...I usually ask what they are doing and try to steer them away from using a lot of scented products or other over the counter products or even other medical products that are perhaps, not appropriate for the condition (GP3, female).

\section{Psychosocial impact on women's lives}

Many GPs acknowledged the psychosocial impact of BV on women, however, those with an interest in sexual health or who saw a higher case-load of female patients of reproductive age showed higher levels of empathy. 
... certainly appreciate that for each individual woman it can create a lot of problems...So some women I've had in tears, because the bacterial vaginosis is getting them down so much, and other women don't seem to be too troubled about the diagnosis (GP1, female).

Only one GP did not think that BV had any psychosocial impact at all, and felt that women experiencing adverse psychosocial impacts must have pre-existing mental health issues as it is a trivial physical condition.

...If it is affecting them mentally or emotionally to the point that it's overtaking their life, there's often an underlying mental health issue anyway (GP4, female).

\section{GPs' actual practice}

GPs' approaches to the diagnosis, management and treatment of BV were varied, inconsistent and considered suboptimal by current STI management guideline standards.

\section{Not using Amsel criteria}

Interestingly, none of the GPs used Amsel criteria to diagnose $\mathrm{BV}$ and most had not even heard of it. Only one reported she vaguely recalled its name.

\section{Slightly rings a bell...but, I don't remember (GP7, female).}

When told about Amsel criteria and that its use is recommended, some GPs thought the criteria were too specific for BV and expressed doubts about its value.

Perhaps if you did it and the $\mathrm{pH}$ indicated that maybe it's bacterial vaginosis, you still gonna want to look at the other potential causes, STIs which are quite concerning, like chlamydia and gonorrhoea... (GP6, male).

One GP was unsure about how to use Amsel criteria in the clinic.

I don't know the practicalities of it actually, like how would you get discharge on a slide and then you use the urine dipstick or something like that (GP7, female).

Other GPs did not have the resources needed for Amsel criteria such as $\mathrm{pH}$ paper, microscopy slides or potassium hydroxide solution.

...so I asked for potassium hydroxide, I asked for $\mathrm{pH}$ paper on one occasion, didn't get them... (GP3, female).

\section{Actual diagnostic approach}

GPs varied in how they diagnosed BV. Half of the GPs - particularly those with an interest in sexual health or who saw higher numbers of female patients of reproductive age - were more thorough in their work-up. They took a sexual history, performed physical examination (including sterile speculum examination) and took a HVS. Others were less thorough, either bypassing the physical examination, or going straight to taking a vaginal swab to diagnose BV.

Have a look, swab it. Quite often you know that's what it is but I'll swab it anyway (GP4, female).

\section{Treatment}

GPs' practices in BV treatment varied. While a couple of GPs reported that they would treat empirically with metronidazole based on clinical suspicion of BV, some preferred to wait for swab results.

I'd defer committing somebody to antibiotics until I've got the swab results (GP5, female).
Other GPs felt inexperienced in managing recurrent BV and either consulted colleagues regarding treatment, looked up guidelines or referred.

...this is quite tricky and I don't have much experience in it. I think if it's recurrent I tend to refer them on to see whether they can be helped by the gynaecologist or by the sexual health physician (GP2, male).

\section{Barriers to care}

Four main barriers were identified in BV management: lack of time, lack of knowledge, training and guidelines, and gender.

\section{Time}

Time was by far the most commonly cited barrier to improving the management of BV in general practice. Many GPs reported limited consultation time and patients often presenting with several issues within a 15-minute consult. One GP felt time limitations would prevent use of the Amsel criteria even if she had resources to do so.

...there's a lot you need to do in the consult, look into their sexual health history, look at other causes of vaginal discharge, check the gynaecology history that sort of thing, so I feel like the Amsel criteria will take a lot of time in clinic when you have to go through sort of a full history and examination anyway... (GP7, female).

\section{Lack of knowledge, training and guidelines}

Another common barrier was GPs' lack of BV knowledge, training and guidelines.

...maybe if I was 10 years out from fellowship, you're not as up to date with your knowledge (GP1, female).

Most GPs had minimal formal training in medical school in relation to $\mathrm{BV}$.

.. wasn't much formal training in medical school or during GP training, but it's something you frequently see clinically (GP1, female).

To learn about BV, GPs relied on job exposure or learning from external sources such as peers, guidelines, journals, or attending additional training in sexual health.

...you learn on the job, you ask questions, you know like any registrar in any training course, by doing it and coming across it (GP4, female).

One GP with a particular interest in sexual health mentioned that she sought additional exposure to BV cases.

...I went out of my way to expose myself to additional training in sexual health at places like [sexual health service]... a lot of the way that general practitioners educate themselves is self-directed so that relies on the practitioner identifying their learning needs by the use of a learning planner, and then meeting those learning needs by undertaking a specific learning ctivity (GP5, female).

\section{Gender}

Lastly, male GPs reported some levels of discomfort in dealing with female sexual health issues. Despite two of the three male GPs indicating in the demographic questionnaire that they were comfortable discussing sexual health issues with female patients, in the interview itself, they presumed female patients with sexual health issues would be more appropriately seen by a female partitioner or women would be more comfortable seeing a female practitioner. 
When they do mention gynaecology symptoms, sexual health symptoms...I raise that with them and then give them the option of seeing the female doctor... (GP8, male).

One GP reported generally feeling comfortable discussing sexual health issues with female patients unless the patient was of similar age

...sometimes I find a little bit challenging is if I have a female patient who's a similar age to me, you know discussing sexual health, can be a little bit uncomfortable... (GP6, male).

Male GPs without an interest in sexual health saw fewer female patients of reproductive age and had fewer opportunities for exposure to BV, likely affecting their knowledge and confidence in BV management.

... I don't see much of it and I don't have that much confidence in my knowledge of women's health presentations (GP8, male).

Further quotes from GPs relating to the main themes are outline in Table 2 .

Table 2. Further GP quotes relating to the four major themes.

\begin{tabular}{|c|c|}
\hline Views \& knowledge & \\
\hline Not medically serious & $\begin{array}{l}\text {...in terms of seriousness it's not going to kill anyone so it's } \\
\text { not, you know, serious in those terms (GP5, female). } \\
\text {..I don't think it's necessarily medically serious (GP8, } \\
\text { male). }\end{array}$ \\
\hline Psychosocial impact & $\begin{array}{l}\text { I think it's quite distressing for women, and I don't take it } \\
\text { lightly from that point of view (GP5, female). } \\
\text { I guess it is something that impacts them quite a lot in terms } \\
\text { of sort of relationships and how they feel about themselves } \\
\text { (GP7, female). } \\
\text { I think it's fairly significant, in that it disrupts quality of life } \\
\text { (GP2, male) }\end{array}$ \\
\hline Actual practice & $\begin{array}{l}\text {...my diagnostic approach will be to take a vaginal swab, } \\
\text { high vaginal swab, either collected by myself or self- } \\
\text { collected by the woman... (GP8, male). } \\
\text { Well usually after sort of taking history, and examination, } \\
\text { checking if they've had pap smears, if they're presenting } \\
\text { solely with vaginal discharge and sort of no other issues I'll } \\
\text { usually do a high vaginal swab, and send that off for } \mathrm{m} / \mathrm{c} / \mathrm{s} \\
\text { (GP7, female). } \\
\text {... at the moment I just take swabs then I often treat } \\
\text { empirically (GP3, female). }\end{array}$ \\
\hline Barriers to care & $\begin{array}{l}\text { I think sometimes we have to admit there is also an element } \\
\text { of sort of not wanting to do it (physical examination) } \\
\text { because it takes so much time, so sometimes even when I } \\
\text { think it could possibly be necessary, it's easy to convince } \\
\text { myself that it's not because of the time constraints (GP2, } \\
\text { male). } \\
\text {..the main barrier is probably the old barrier for everything } \\
\text { which is time, you know, the BEACH data shows that GPs } \\
\text { are increasingly expected to do more with less you know, } \\
\text { barely a week goes past when you don't use something like } \\
\text { GPs should be weighing everybody GPs and I think hang on, } \\
\text { so there's another thing we're meant to do in addition to the } \\
\text { reason the patient comes in, you know we should be } \\
\text { checking on this and that and whatever so on (GP4, female). } \\
\text { Ithink the main barrier, the fact that it's not regarded as a } \\
\text { thing you know. Everyone knows about thrush but very few } \\
\text { people know about BV, so I think it's more of an education } \\
\text { problem in practice... (GP3, female). }\end{array}$ \\
\hline
\end{tabular}

\section{Discussion}

Many GPs in this study did not consider BV to be a serious condition associated with adverse physical complications in nonpregnant women. Notably, most were unaware of the increased risk of HIV and STIs and overall lacked knowledge around sexual and lifestyle risk factors. Whilst the majority of GPs acknowledged BV had some impact on women's quality of life, those with an interest in sexual health tended to show higher levels of empathy. Overall, GPs reported minimal formal training around BV in medical school and observed that learning was often self-directed and 'on the job'. Despite guidelines recommending otherwise, no GP used the Amsel criteria to diagnose $\mathrm{BV}$, and diagnostic and treatment practices were often inconsistent and considered suboptimal by current STI management guideline standards.

In Australia, medical schools are expected to meet generic competencies specified by the Australian Medical Council [21)] These do not indicate particular proficiencies in sexual and reproductive medicine. Medical school training in sexual and reproductive health is variable and dependent on each university. It could be expected that GPs without a particular interest in sexual health are less exposed to and thus knowledgeable about BV. However, this study found that whether or not GPs had a stated interest in sexual health, overall knowledge around associated adverse physical sequelae and risk factors of BV was lacking, a finding which needs further exploration with larger sample.

Similar to previous studies conducted in the US and UK [13-16,22], our study found BV knowledge, as well as diagnostic and management practices, were suboptimal. Lack of formal education or training might contribute to poor levels of knowledge, which could then translate into suboptimal clinical practices. While it is tempting to recommend that more formal BV training be included in the GP curriculum, the reality is that GPs already have a vast range of medical conditions to learn as part of their syllabus. Other changes in women's health such as the revised National Cervical Screening Program are rightly likely to take precedence over increased BV education.

Time is the other major barrier. While Australian data exploring the barriers faced by GPs regarding BV management is lacking, prior sexual health studies have consistently shown time to be a major barrier $[23,24]$. Given these time constraints, it follows then that some GPs in our study found it challenging to be thorough in their work-up and management of BV. Gender barriers to discussing sexual health, be it women not feeling comfortable speaking to male GPs or vice versa, have also been identified in previous studies [25,26], and meant that male GPs often had less exposure to BV cases, potentially leading to deskilling in BV diagnosis and management Many issues raised by women around the management of BV in Australian general practice have also been identified to varying degrees in this study - GPs' lack of knowledge, substandard diagnostic approaches and inconsistencies in management [18]. However, while women have previously reported a lack of sensitivity and recognition of the impact of BV [18], most GPs in this study did recognise to some degree, the impact BV can have on women's lives. It is possible there is a discrepancy between how women would like GPs to recognise and acknowledge the impact BV, and the degree to which GPs think they have.

\section{Strengths and limitations}

The main limitation of this study was that as a six-month final year medical student project, recruitment time was finite which impacted 
on our capacity to meet data saturation. While data saturation was evident around some themes, including the seriousness of BV, poor knowledge levels and a lack of training, additional interviews would have been beneficial for a deeper understanding and exploration of GPs diagnostic and treatment approaches, barriers to care and to allow for intergroup comparisons i.e. by gender and practice location.

The major strength of this study is that it is the first Australian study to explore GPs' knowledge and practices around BV management. This study adds to very limited data available in this area and further understanding of GPs' views and practices in the management of bacterial vaginosis.

\section{Implications for General Practice}

- The management of BV in general practice could be enhanced through a better understanding of BV, including well-known risk factors and adverse physical sequelae.GPs should ask women about the use of self-help remedies as they may be exacerbating symptoms or recurrences.

- GPs should offer women evidence-based advice around the risks associated with BV and optimal ways to manage BV.

- While GPs may not consider BV a serious condition, a sensitive attitude and acknowledgement of the impact symptoms can have on women's lives would go a long way toward addressing women's concerns about BV management in general practice.

- A dedicated website to improve women and healthcare professional's awareness of BV and provide clear, credible information, resources and evidence based advice would likely be of benefit.

\section{Acknowledgements}

We would like to thank all the GPs who kindly consented to participate in our study.

\section{Conflict of Interest}

The authors declare no conflict of interest

\section{Funding}

Dr Jade Bilardi is in receipt of an NHMRC Early Career Fellowship No 1013135.

\section{References}

1. Allsworth JE, Peipert JF (2007) Prevalence of bacterial vaginosis: 2001-2004 National Health and Nutrition Examination Survey data. Obstet Gynecol 109: 114-120. [Crossref]

2. Bradshaw CS, Walker J, Fairley CK, Chen MY, Tabrizi SN, et al. (2013) Prevalent and incident bacterial vaginosis are associated with sexual and contraceptive behaviours in young Australian women. PLoS ONE 8: e57688.

3. Fethers KA, Fairley CK, Hocking JS, Gurrin LC, Bradshaw CS (2008) Sexual risk factors and bacterial vaginosis: a systematic review and meta-analysis. Clin Infect Dis 47: 1426-1435.

4. Klebanoff MA, Nansel TR, Brotman RM, Zhang J, Yu K-F, et al. (2010) Personal hygienic behaviors and bacterial vaginosis. Sex Transm Dis 37: 94.

5. Hillier SL, Nugent RP, Eschenbach DA, Krohn MA, Gibbs RS, et al. (1995) Association between bacterial vaginosis and preterm delivery of a low-birth-weight infant. The Vaginal Infections and Prematurity Study Group. N Engl J Med 333: 1737-1742.

6. Ralph SG, Rutherford AJ, Wilson JD (1999) Influence of bacterial vaginosis on conception and miscarriage in the first trimester: cohort study. BMJ 319: 220-223. [Crossref]
7. Brotman RM, Klebanoff MA, Nansel TR, Yu KF, Andrews WW, et al. (2010) Bacterial vaginosis assessed by gram stain and diminished colonization resistance to incident gonococcal, chlamydial, and trichomonal genital infection. J Infect Dis 202: 19071915. [Crossref]

8. Myer L, Kuhn L, Stein ZA, Wright TC, Denny L (2005) Intravaginal practices, bacterial vaginosis, and women's susceptibility to HIV infection: epidemiological evidence and biological mechanisms. Lancet Infect Dis 5: 786-794.

9. Bilardi JE, Walker S, Temple-Smith M, McNair R, Mooney-Somers J, et al. (2013) The burden of bacterial vaginosis: women's experience of the physical, emotional, sexua and social impact of living with recurrent bacterial vaginosis. PLOS ONE 8: e74378.

10. Australasian Society for HIV (2018) Viral Hepatitis and Sexual Health Medicine. Australian STI Management Guidelines For Use In Primary Care. Vaginal discharge.

11. Australasian Society for HIV (2018) Viral Hepatitis and Sexual Health Medicine. [Online] Australian STI Management Guidelines For Use In Primary Care. Bacterial Vaginosis.

12. Bornstein J, Lakovsky Y, Lavi I, Bar-Am A, Abramovici H (2001) The classic approach to diagnosis of vulvovaginitis: a critical analysis. Infect Dis Obstet Gynecol 9: 105-111. [Crossref]

13. Anderson MR, Karasz A (2005) How do clinicians manage vaginal complaints? An Internet survey. MedGenMed 7: 61.

14. Langsford MJ, Dobbs FF, Morrison GM, Dance DA (2001) The effect of introduction of a guideline on the management of vaginal discharge and in particular bacterial vaginosis in primary care. Fam Pract 18: 253-257.

15. Schwiertz A, Taras D, Rusch K, Rusch V (2006) Throwing the dice for the diagnosis of vaginal complaints? Ann Clin Microbiol Antimicrob 5: 4.

16. Wiesenfeld HC, Macio I (1999) The infrequent use of office-based diagnostic tests for vaginitis. Am J Obstetand Gynecol 181: 39-41.

17. Karasz A, Anderson M (2003) The vaginitis monologues: women's experiences of vaginal complaints in a primary care setting. Soc Sci Med 56: 1013-1021.

18. Bilardi J, Walker S, McNair R, Mooney-Somers J, Temple-Smith M, et al. (2016) Women's management of recurrent bacterial vaginosis and experiences of clinical care: A qualitative study. PLOS ONE 11: e0151794.

19. Neergaard MA, Olesen F, Andersen RS, Sondergaard J (2009) Qualitative description the poor cousin of health research? BMC Med Res Methodol 9: 52. [Crossref]

20. Grbich C (2007) General approaches to designing and analyzing data. Qualitative data analysis: An introduction. Sage Publications, London, UK.

21. Australian Medical Council (2018) Australian Medical Council Limited.

22. Callahan DB, Weinberg M, Gunn RA (1999) Bacterial vaginosis in pregnancy: diagnosis and treatment practices of physicians in San Diego, California. Sex Transm Dis 30: 645-649.

23. Hocking JS, Parker RM, Pavlin N, Fairley CK, Gunn JM (2008) What needs to change to increase chlamydia screening in general practice in Australia? The views of general practitioners. BMC Public Health 8: 425.

24. Temple-Smith M, Hammond J, Pyett P, Presswell N (1996) Barriers to sexual history taking in general practice. Aust Fam Physician 25: S71- S74.

25. Dyer K, das Nair R (2013) Why don't healthcare professionals talk about sex? A systematic review of recent qualitative studies conducted in the United Kingdom. $J$ Sex Med 10: 2658-2670.

26. Gott M, Galena E, Hinchliff S, Elford H (2004) "Opening a can of worms": GP and practice nurse barriers to talking about sexual health in primary care. Fam Pract 21: 528-536.

Copyright: (C2018 Toh J. This is an open-access article distributed under the terms of the Creative Commons Attribution License, which permits unrestricted use, distribution, and reproduction in any medium, provided the original author and source are credited. 